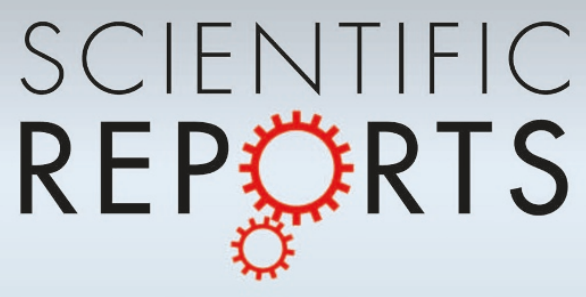

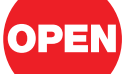

SUBJECT AREAS:

MESENCHYMAL

MIGRATION

CELL ADHESION

ONCOGENESIS

EXPERIMENTAL MODELS OF

DISEASE

Received

25 October 2012

Accepted

27 December 2012

Published

28 January 2013

Correspondence and requests for materials should be addressed to

S.T. (toyokuni@med. nagoya-u.ac.jp)

\section{Metamorphosis of mesothelial cells with active horizontal motility in tissue culture}

\author{
Hirotaka Nagai' ', Shan Hwu Chew', Yasumasa Okazaki', Satomi Funahashi' ${ }^{1}$, Takashi Namba², \\ Takuya Kato ${ }^{3}$, Atsushi Enomoto ${ }^{3}$, Li Jiang', Shinya Akatsuka' \& Shinya Toyokuni'
}

\begin{abstract}
'Department of Pathology and Biological Responses, Nagoya University Graduate School of Medicine, Nagoya, 466-8550, Japan, ${ }^{2}$ Department of Cell Pharmacology, Nagoya University Graduate School of Medicine, Nagoya, 466-8550, Japan, ${ }^{3}$ Department of Tumor Pathology, Nagoya University Graduate School of Medicine, Nagoya, 466-8550, Japan.
\end{abstract}

Mesothelial cells, which have diverse roles in physiology and pathology, constitute the mesothelium along with connective tissue and the basement membrane; the mesothelium serves to shield the somatic cavities. After mesothelial injury, mesothelial cells undergo tissue recovery. However, the mechanism of mesothelial regeneration remains poorly understood. In this study, we used confocal time-lapse microscopy to demonstrate that transformed mesothelial cells (MeT5A) and mouse peritoneal mesothelial cells can randomly migrate between cells in cell culture and in ex vivo tissue culture, respectively. Moreover, peritoneal mesothelial cells changed their morphology from a flattened shape to a cuboidal one prior to the migration. Conversely, MDCKII epithelial cells forming tight cell-cell contacts with one another do not alter the arrangement of adjacent cells during movement. Our evidence complements the current hypotheses of mesothelial regeneration and suggests that certain types of differentiated mesothelial cells undergo morphological changes before initiating migration to repair injured sites.

 he mesothelium, which is a membrane that covers three somatic cavities (pleural, peritoneal and pericardial) and the surface of visceral organs, consists of mesothelial cells, the basement membrane and supporting connective tissue ${ }^{1,2}$. Mesothelial cells are generally flattened with a squamous cell-like appearance. In addition, electron microscopy has revealed numerous microvilli that cover the surfaces of these cells ${ }^{3}$. The physiological functions of mesothelial cells are surprisingly diverse $\mathrm{e}^{1,4-6}$. These cells' main function is to prevent internal organs from adhering to one another. However, mesothelial cells are also involved in immune regulation, coagulation, fibrinolysis, and the transport of fluid and molecules. Although great advances have improved our understanding of mesothelial physiology, many aspects of these cells remain unknown. The current trend of increasing incidence of mesothelioma, which is a malignancy arising from mesothelial cells, demands further insight into the normal physiology of these cells to understand the pathological changes they may undergo ${ }^{7-11}$.

Mesothelial cell injury, which may lead to the development of pleural/peritoneal adhesion, effusion and malignant mesothelioma ${ }^{12-15}$, can occur during medical procedures, such as peritoneal dialysis or surgery, or when the cells are exposed to fibrous particles, such as asbestos fibers. A previous study has reported that injured mesothelial cells are capable of self-recovery. However, there are inconclusive data regarding how the mesothelial cells repair themselves after suffering injuries. A number of studies have suggested that mesothelial cells that surround an injured site proliferate and migrate into the wounded area ${ }^{16,17}$. Previous studies have shown that freefloating mesothelial cells are incorporated into the injured site and repopulate that area ${ }^{18,19}$. Several studies have suggested that mesothelial cells regenerate via differentiation of subserosal progenitor cells, which migrate to the serosal surface ${ }^{20,21}$. One of these proposed mechanisms may be predominant, or all of these different mechanisms might contribute equally to mesothelial recovery following injury. One interesting finding is that following mesothelial injury, many researchers have observed a morphological change in mesothelial cells from a relatively flattened shape to a cuboidal one ${ }^{17,22,23}$. Based on an ultrastructural analysis, these cuboidal, cobblestone-like mesothelial cells with prominently increased cell volume contain abundant mitochondria, increased area of rough endoplasmic reticulum and a well-developed Golgi apparatus, indicating that mesothelial cells display increased metabolic activity after undergoing this morphological change ${ }^{24}$.

Many previous studies have been based on the observation of mesothelial cells after fixation. Although useful, these conventional methods are not able to demonstrate the dynamics of living cells. Indeed, one of the aforementioned mechanisms postulates that mesothelial cells are able to migrate and repopulate a wounded site. However, no direct demonstration of mesothelial cell migration on living tissue has been indicated in these 
a

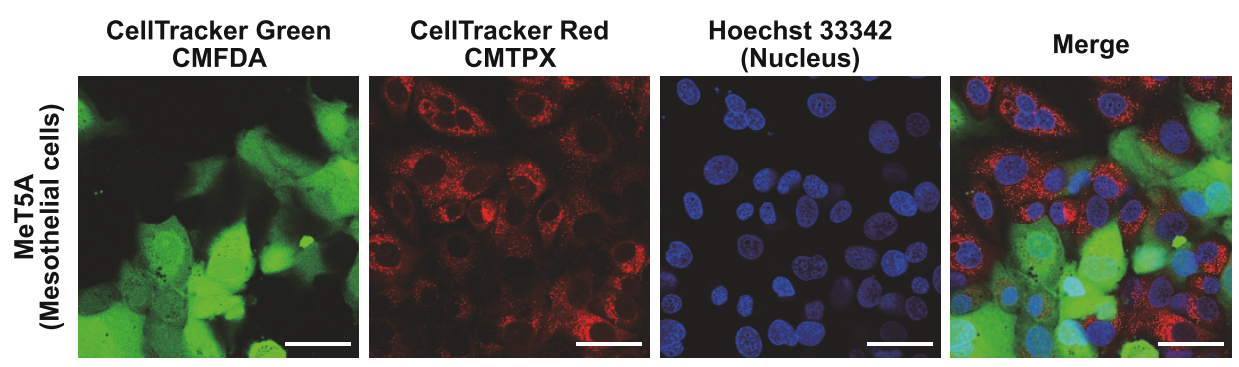

b
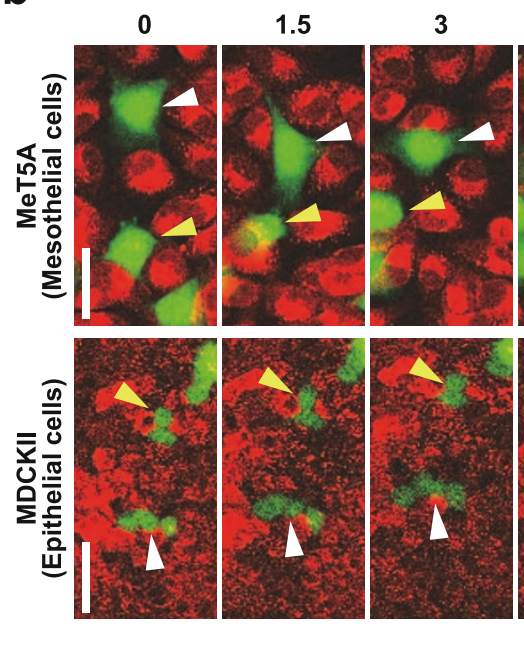

4.5

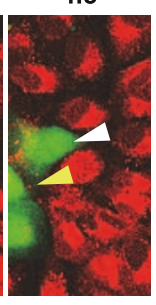

6

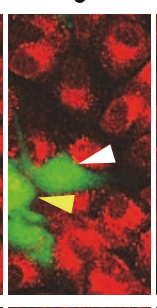

7.5

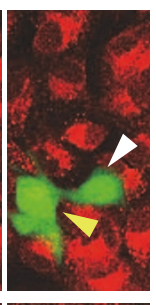

9

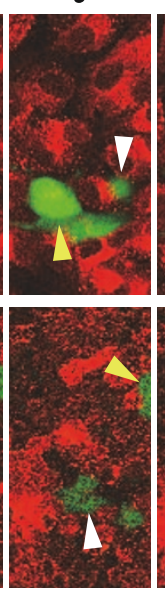

10.5

12 (h)

C
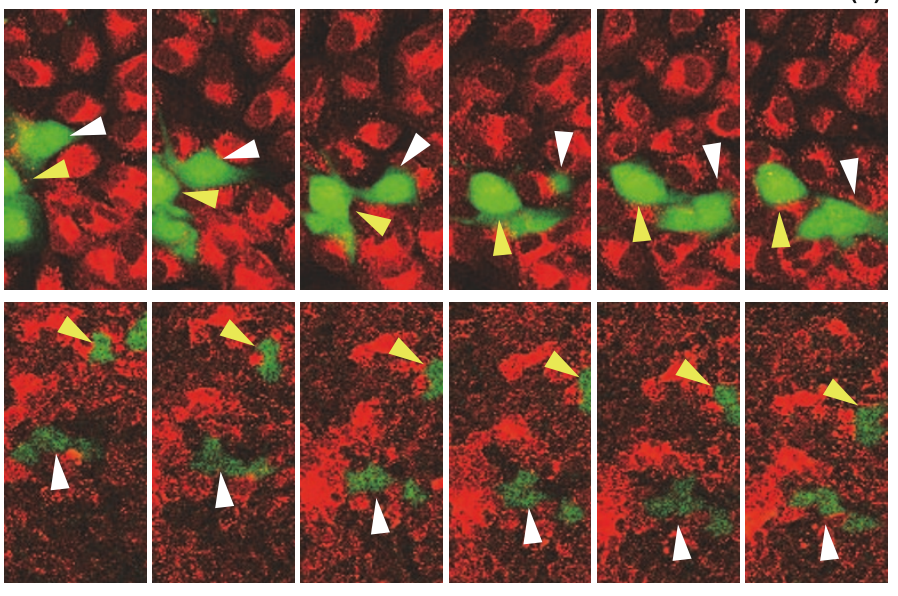

d
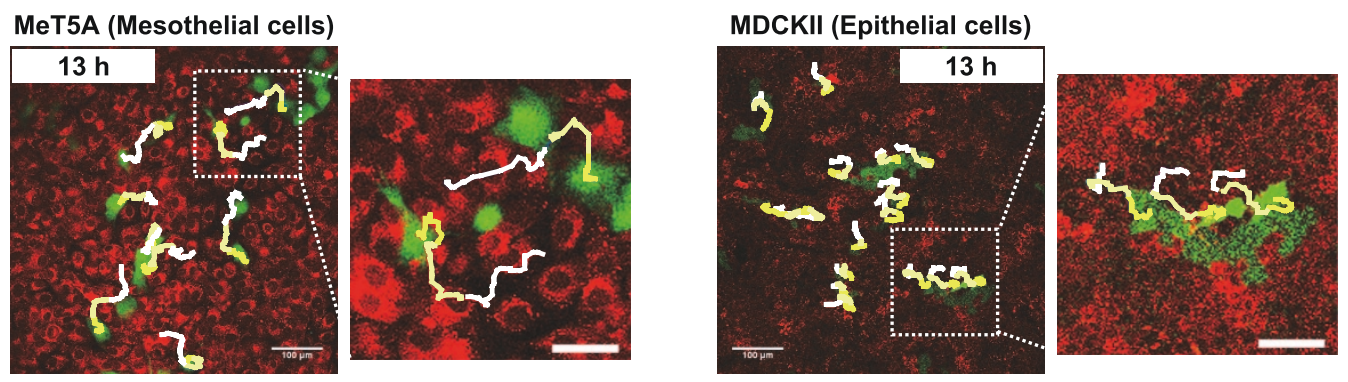

e Location from the origin $(\mu \mathrm{m})$

f
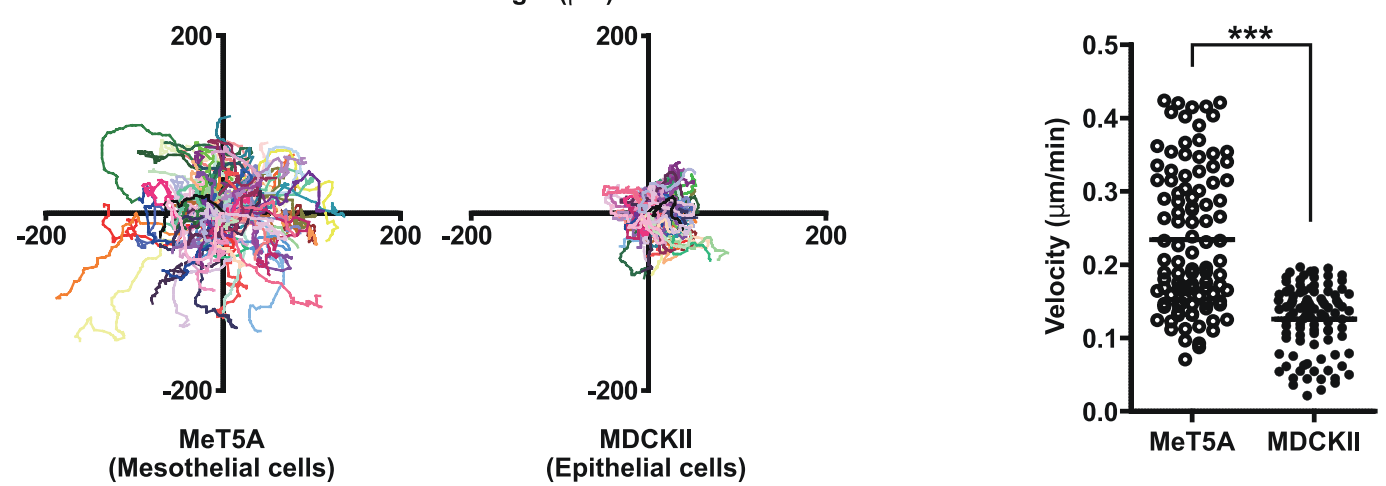

Figure $1 \mid$ Mesothelial cells migrate through adjacent cells. (a) Confirmation of lack of interference of two types of cell tracking reagents (CellTracker Green CMFDA and CellTracker Red CMTPX). We used Hoechst 33342 to stain the cell nuclei. (b) MeT5A mesothelial cells changed places with each other as indicated by the white and yellow arrowheads. MDCKII epithelial cells did not migrate but swayed back and forth. (c) and (d) Manual cell tracking of MeT5A mesothelial cells and MDCKII epithelial cells using time-lapse images that were acquired at 6-min intervals for $13 \mathrm{~h}$. The initial and terminal places of tracked cells were connected using a line with gradation from white (initial) to yellow (terminal). Squares in the top panels are magnified in the bottom panels. Scale bars (a-d): $50 \mu \mathrm{m}$. (e) Cell tracking lines for one hundred cells in three independent experiments for each cell line. The xy coordinates indicate the location from the initial place of each cell. (f) Average velocities of each cell during time-lapse imaging. $* * * p<0.001$. 
a

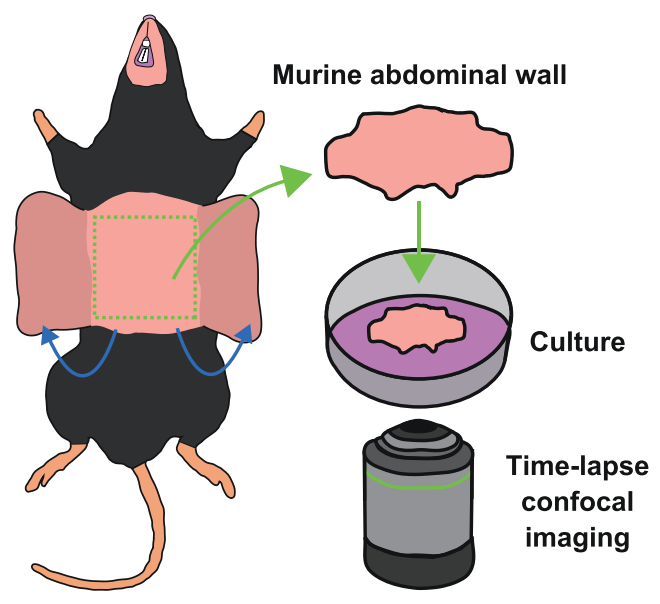

Parietal mesothelial cells on a murine abdominal wall
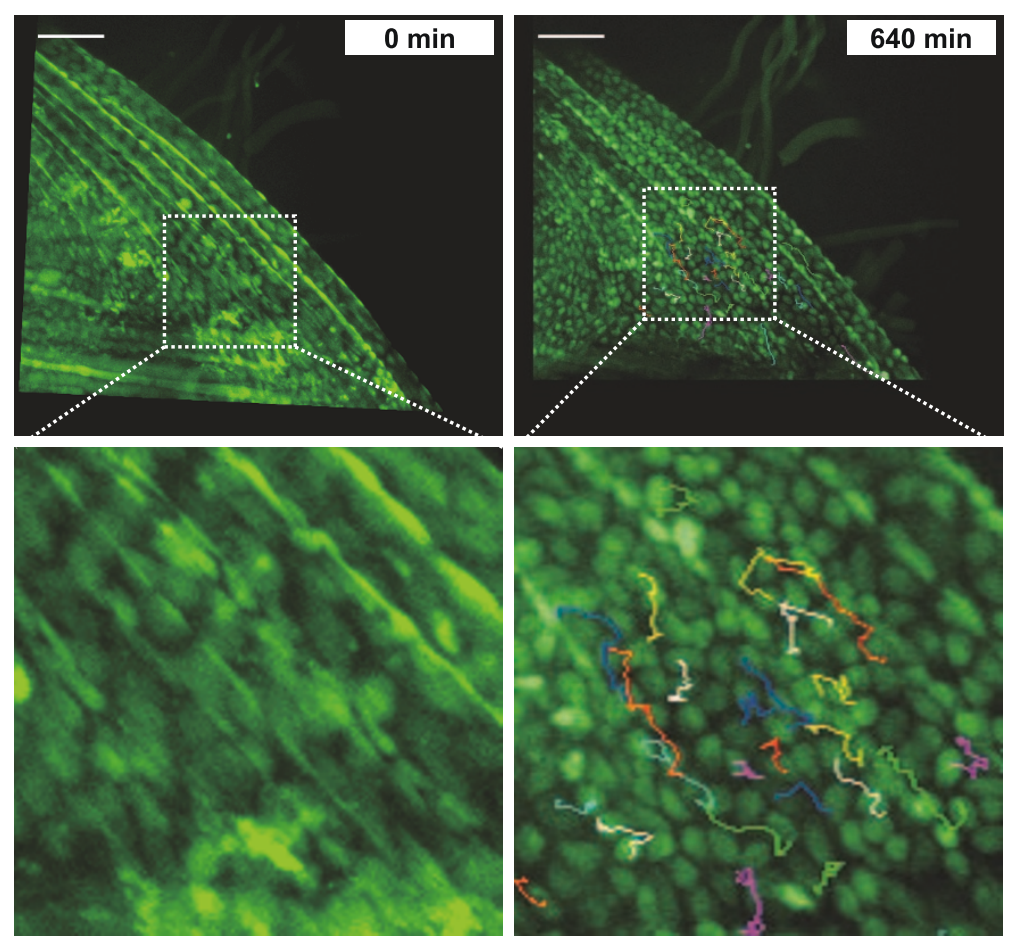

C

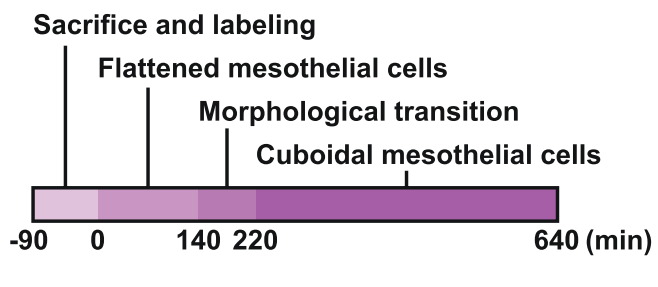

d

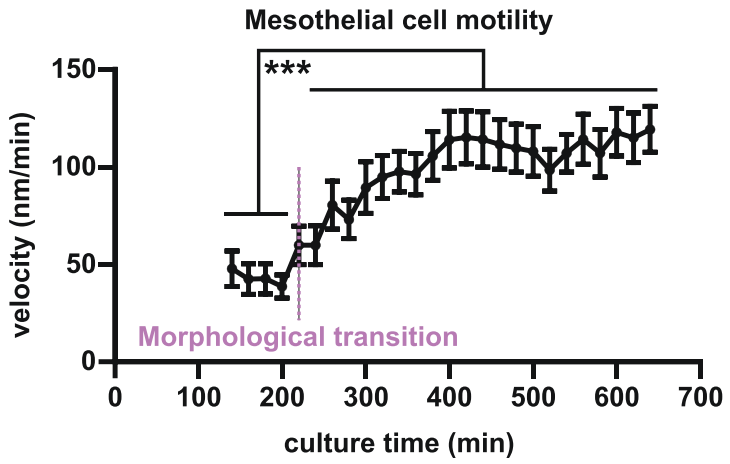

Figure $2 \mid$ Flattened mesothelial cells change their shape into cuboidal cells in cultured murine abdominal wall tissue. (a) A schematic for the current tissue culture method. (b) Flattened mesothelial cells before incubation (left) and cuboidal mesothelial cells after 640 min of incubation (right). The initial and terminal places of tracked cells were connected using a colored line. Squares at the top are magnified at the bottom. Scale bars: $100 \mu \mathrm{m}$. (c) A schematic of the time-course of mesothelial morphology using the current tissue method. (d) Time-course of velocity of mesothelial cells tracked. Thirty or more cells were tracked from each sample. Two independent results $(n=62)$ are shown. ${ }^{* * *} \mathrm{p}<0.001$.

studies. In the current study, we used a novel tissue culture method that was combined with a time-lapse confocal microscopy imaging system to provide supportive evidence for the migration and morphological change of mesothelial cells under conditions that resemble in vivo conditions.

\section{Results}

Mesothelial cells migrate between cells. First, we compared mesothelial cell behavior to epithelial cell behavior in vitro. We used a non-malignant human mesothelial cell line, MeT5A, which had been established by SV40 (simian virus 40) transformation. For comparison, we used the polarized non-transformed canine renal epithelial cell line, MDCKII. To observe cell motility, cultured cells were separately labeled with two types of cell tracking reagents (CellTracker Green CMFDA and CellTracker Red CMTPX). Separately labeled cells were later mixed together and co-cultured.
These cells were seeded at a sub-confluent density to mimic in vivo conditions. The motility of green-labeled cells among the red-labeled cell population was tracked using time-lapse confocal microscopy. As shown in Fig. 1a, the admixed cells showed clear, distinct color labels with no cross-interference. Using confocal microscopy, we demonstrated that MeT5A cells were able to migrate in a horizontal manner by moving between the other surrounding cells. In contrast, the MDCKII cells remained in their original places and did not exhibit further migration other than slightly moving back and forth (Fig. 1b).

To determine the migratory distance of MeT5A cells, we tracked the cells for $13 \mathrm{~h}$. MeT5A cells migrated in various directions to a distant site (Fig. 1c and d), whereas MDCKII cells slightly moved back and forth without changing their arrangement with neighboring cells. The quantitative data of cell motility are shown in Figs. 1e and $\mathrm{f}$. We calculated the velocity of movement based on the location 


\section{Abdominal wall surface}

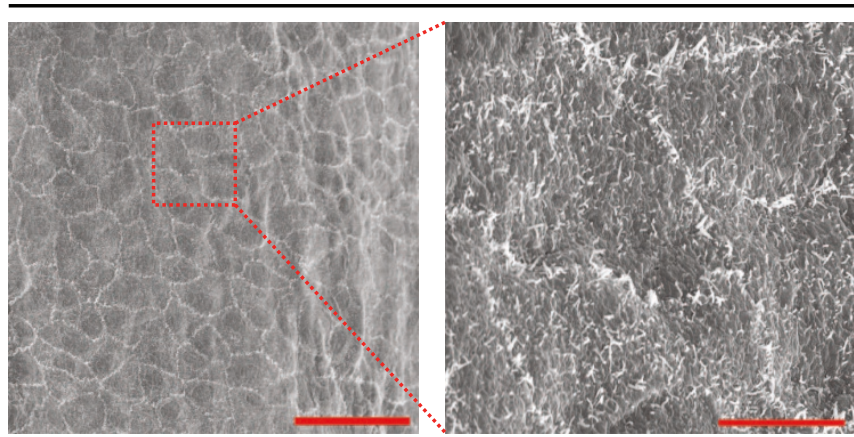

Before culture

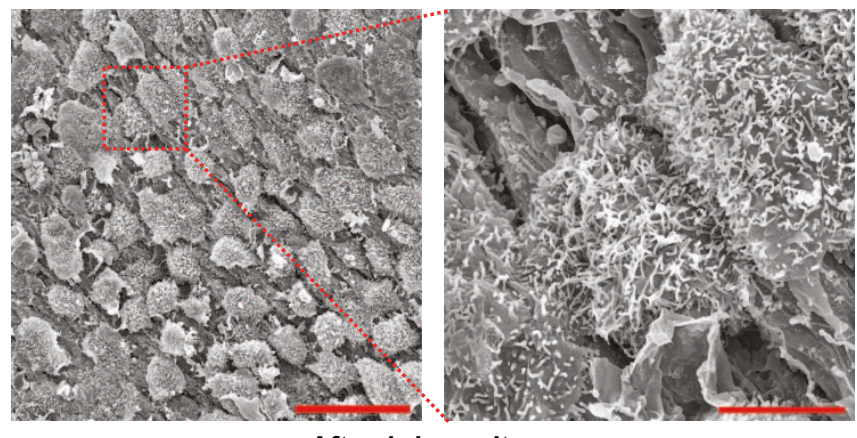

After 1 day culture

Figure $3 \mid$ The morphological change of mesothelial cells after tissue culture. Scanning electron microscopic images of the surface of murine abdominal walls before and after tissue culture for 1 day. Scale bars: $30 \mu \mathrm{m}$.

from the original position. MeT5A cells migrated a longer distance at a sub-confluent density, and their velocity of movement was also significantly higher compared to the migratory distance and velocity of the MDCKII cells.

Flattened mesothelial cells change their shape into cuboidal cells and start to horizontally migrate in tissue culture. After observing the behavior of mesothelial cells that were cultured in vitro, we determined whether mesothelial cells exhibited similar behaviors under ex vivo conditions. We established a simple but novel approach to observe the behavior of mesothelial cells ex vivo. First, we excised the anterior abdominal wall from a mouse and labeled the mesothelial cells that covered the surface of the abdominal wall with CellTracker Green CMFDA. The abdominal wall tissue was subjected to time-lapse confocal microscopy imaging (procedure summarized in Fig. 2a). As shown in Fig. 2b (left panel), the surface of abdominal wall was lined with a monolayer of flattened mesothelial cells when the observation began. However, cuboidalshaped mesothelial cells were clearly observed in the images that were captured approximately $11 \mathrm{~h}$ later (Fig. 2 b, right panel). This result indicates that at least a portion of the mesothelial cells undergo a morphological transition from a flattened to cuboidal shape. This morphological change began at approximately $140 \mathrm{~min}$ after the initial time point (Fig. 2c and Movie S1). More importantly, these cuboidal mesothelial cells exhibited migratory behaviors that were identical to those observed with Met5A mesothelial cells in vitro. We measured the velocity of the mesothelial cells and confirmed that the cells started to migrate horizontally between the surrounding cells after morphological transition (Fig. $2 \mathrm{~b}$ and $\mathrm{d}$ and Movie S1).

We confirmed that mesothelial cells horizontally migrated ex vivo and in vitro. To corroborate the finding of mesothelial morphological changes in tissue culture, we used a scanning electron microscopy. Consistent with the aforementioned results, the mesothelial cells exhibited a cuboidal shape after 1 day of culture (Fig. 3). The significance of these morphological changes in mesothelial cell migration requires further study.

Finally, to validate our ex vivo culture system, we evaluated the mesothelial cells by various methods. First, to exclude the possibility that mesothelial cells became cuboidal because of being apoptotic, we performed TUNEL staining. After $16 \mathrm{~h}$ of tissue culture, abdominal muscles were apoptotic (Fig. 4a, solid arrows). However, mesothelial cells did not show apoptosis (Fig. 4a, dotted arrows) compared with the positive control. We also confirmed that there were no apoptotic cells before starting tissue culture. Second, to investigate cultureassociated damages in mesothelial cells, we evaluated the hypoxic state of the cells using a pimonidazole-based assay. This small chemical forms protein adducts in hypoxic cells; thus, a particular primary antibody against pimonidazole-adducts recognizes the cells under hypoxia. Because this chemical needs incubation time, we added pimonidazole to tissue culture medium immediately after and $14 \mathrm{~h}$ after tissue culture started, which in total corresponds to $2 \mathrm{~h}$ and $16 \mathrm{~h}$ of incubation, respectively. After fixing the tissue, we performed immunohistochemistry using anti-pimonidazole-adducts antibody. We measured the fluorescence intensity of mesothelial cells and found that mesothelial cells were slightly hypoxic after $16 \mathrm{~h}$ culture compared with $2 \mathrm{~h}$ culture (Fig. $4 \mathrm{~b}$ and $\mathrm{c}$ ). Although this slight hypoxia did not lead to mesothelial apoptosis, the hypoxia may have contributed to the morphological transition of the mesothelial cells.

\section{Discussion}

Mesothelial cells perform a plethora of diverse cellular functions. However, the normal physiology (e.g., motility, proliferation and differentiation) of these cells is not thoroughly understood ${ }^{1,2}$. Although several previous studies have suggested that mesothelial cells undergo morphological transitions in response to inflammation or mesothelial injury ${ }^{17,22,23}$, there has been a lack of direct evidence regarding this morphological change, especially in a setting that resembles in vivo conditions. This discrepancy might be due to the unavailability of an experimental approach that allows direct observation of the dynamics of mesothelial cell behavior.

In this study, we described a simple but novel technique that was established to observe the physiological changes in mesothelial cells. Using our cultured-tissue method, we provided direct evidence of the morphological transition in mesothelial cells without cell death (i.e., apoptosis). Using confocal microscopy imaging, we observed that mesothelial cells within the mouse abdominal wall tissue morphologically changed from a flattened shape to a cuboidal one. Although the altered cells were not facing the injured site, these cells horizontally migrated across the tissue by squeezing themselves through the gaps of surrounding cells. These ex vivo results are in accordance with those obtained using the cultured human mesothelial cell line, MeT5A. We found that the mesothelial cells migrated a greater distance compared to the epithelial cells. Therefore, our findings confirm the previous hypothesis that mesothelial cells undergo morphological changes and subsequent migration following injury.

Indeed, the morphological changes in mesothelial cells following injury have been recognized for many decades. Previous findings have suggested that mesothelial cells that directly face an injured site migrate to cover the wounded area ${ }^{16,17}$. We hypothesized that two possible factors are involved in mesothelial cell changes in morphology and motility: the loss of cell-cell interactions and the release of soluble factors. It is a well-established fact that cell-cell interaction is closely related to the regulation of cell polarity, motility and morphology, typically via cadherin-mediated signaling pathways ${ }^{25,26}$. As damage to mesothelial cells is inevitably accompanied by loss of cellcell interaction due to cell death, such loss of cell-cell interaction seems to be a reasonable explanation for the initiation of morphological changes in mesothelial cells. However, based on the present results, we cannot explain the induction of morphological changes and motility in mesothelial cells by loss of cell-cell interaction alone. 
a

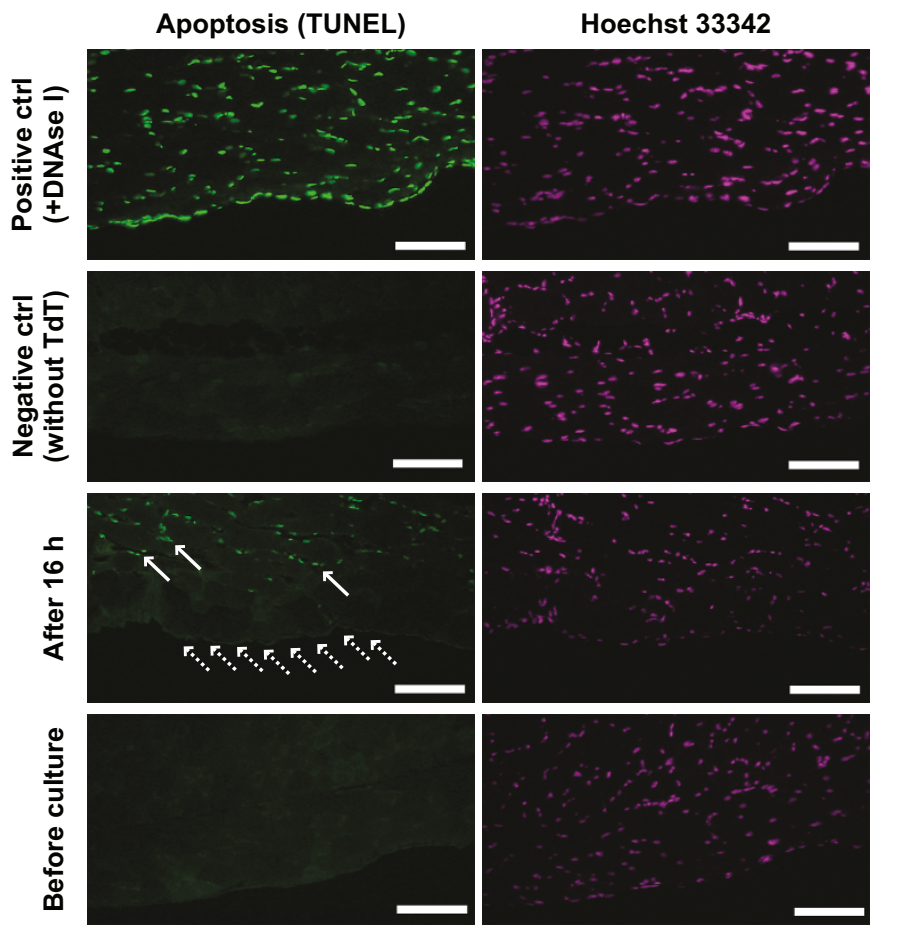

b

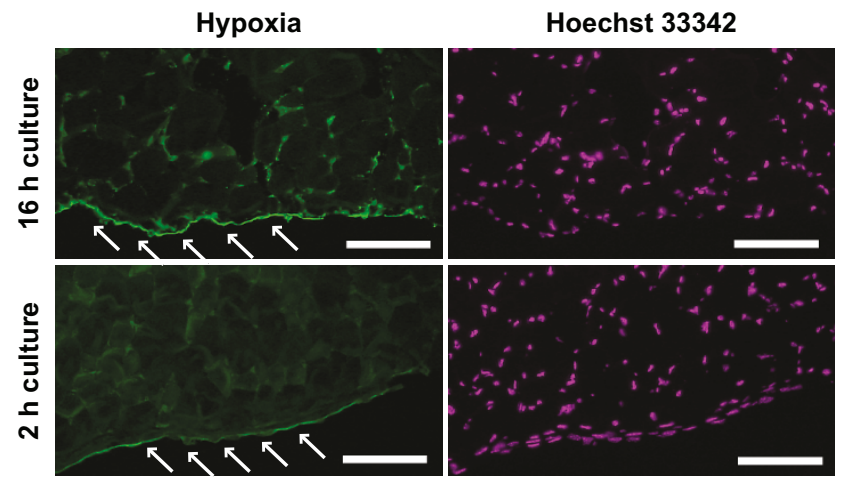

C

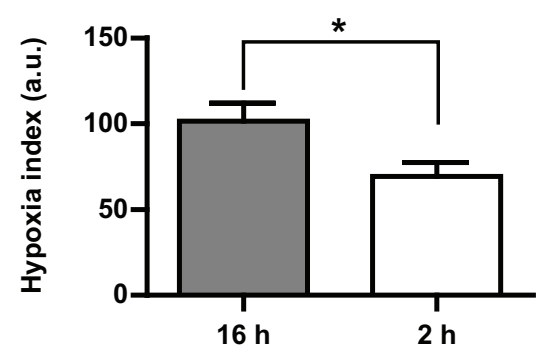

Culture duration

Figure $4 \mid$ Mesothelial cells were not apoptotic but slightly hypoxic after tissue culture. (a) Green fluorescence indicates apoptotic or digested-DNA. After $16 \mathrm{~h}$ of culture, muscle cells exhibit apoptosis (arrows), but mesothelial cells do not (dotted arrows). Before tissue culture, both muscle and mesothelial cells show no sign of apoptosis. Hoechst 33342 indicates nuclei. Mesothelial cells were distinguished from muscle cells for their flattened morphology and localization limited to the parietal surface of the abdominal wall. (b) Green fluorescence shows the abundance of pimonidazole-adducts, which reflects the extent of hypoxia (refer to text for details). After $16 \mathrm{~h}$ of tissue culture, mesothelial cells are slightly hypoxic (top left, arrows) compared with 2-h culture condition (bottom left, arrows). Scale bars (a and b): $100 \mu \mathrm{m}$. (c) Intensity of green fluorescence in panel (b) was measured with ImageJ. As-measured-intensity of fluorescence in the range 0 to 255 is shown in an arbitrary unit. ${ }^{*} \mathrm{p}<0.05$.

We observed morphological transitions followed by migration in mesothelial cells, which were not situated immediately adjacent to the injured site.

Therefore, our evidence is consistent with the hypothesis that morphological changes in mesothelial cells are induced by putative soluble factors because mesothelial cells going through the conversion retain adjacent cells. However, what types of factors are involved is still elusive. Several previous studies have investigated the molecular mechanisms that regulate normal mesothelial cell behavior and have provided clues. Hott et al. have reported that thrombin enhances proliferation and migration of rat pleural mesothelial cells ${ }^{27}$. Narsreen et al. have demonstrated that MCP-1, which is secreted by macrophages, induces proliferation and haptotaxis of human pleural mesothelial cells via the MCP-1/CCR2 signaling pathway $^{28}$. Therefore, thrombin- and MCP-1-mediated inflammation may contribute to mesothelial migration ${ }^{29,30}$. A previous study has reported that mesothelial cells secrete IL-6 and IL-8 during inflammation $^{31,32}$ and that these cytokines might be involved in a positive feedback loop that results in the activation of mesothelial cells. Necrotic factors also activate inflammatory responses in the mesothelial cells (i.e., activation of NF- $\kappa \mathrm{B}$ and secretion of CXCL1 and interleukin-6) ${ }^{33}$. These factors represent potentially important candidates that may induce morphological changes and the subsequent migration of mesothelial cells after tissue damage. However, it is highly likely that other factors might be involved. Specifically, in the present study, hypoxia might also have contributed to the mesothelial changes. Therefore, further investigation is certainly necessary to characterize these factors. We expect that the identification of molecules that initiate or terminate the morphological and migratory changes will improve our understanding of mesothelial physiology and pathology.

In conclusion, we described a novel tissue culture approach for the observation of the dynamic behavior of mesothelial cells. This methodology was useful to evaluate mesothelial morphology and motility in conditions that resembled physiological settings compared to using an in vitro model. Using this approach, we provided supportive evidence that after injury, mesothelial cells undergo morphological changes and migrate to the injured area from a distant site. We hypothesize that these processes are induced by soluble factors that are released from damaged cells. Further investigation is required to identify the factors that comprise the key elements to this migratory event.

\section{Methods}

Materials. We obtained MeT5A cells from the American Type Culture Collection (Manassas, VA, USA). The MDCKII cells were a generous gift from Dr. Atsushi Enomoto (Nagoya University). C57BL/6 mice were purchased from Charles River Laboratories Japan, Inc. The Animal Experiment Committee of the Nagoya University Graduate School of Medicine approved the animal experiments. We purchased CellTracker Green CMFDA (Invitrogen), CellTracker Red CMTPX (Invitrogen), Hoechst 33342 (Dojindo Laboratories, Kumamoto, Japan), Hypoxyprobe $^{\mathrm{TM}}-1 \mathrm{Kit}$ (Hypoxyprobe, Inc., Burlington, MA) and collagen (Cellmatrix Type I-C; Nitta Gelatin, Inc., Osaka, Japan).

Cell culture conditions. MeT-5A cells were maintained in Medium 199 (Invitrogen) that was supplemented with $1 \%$ antibiotic-antimycotic solution (Invitrogen), $10 \mathrm{ng} / \mathrm{ml}$ epidermal growth factor, $400 \mathrm{nM}$ hydrocortisone, $870 \mu \mathrm{M}$ insulin, $0.3 \%$ Trace Elements B (Mediatech, Manassas, VA, USA) and 10\% FBS-Gold (PAA Laboratories, Ltd.). MDCKII cells were cultured in DMEM that was supplemented with $1 \%$ antibiotic-antimycotic solution and $10 \%$ FBS-Gold. All of the cells were maintained at $37^{\circ} \mathrm{C}$ with $5 \% \mathrm{CO}_{2}$. 
Time-lapse imaging of sub-confluent cells in vitro. MeT5A and MDCKII cells were seeded at 30,000 cells $/ \mathrm{cm}^{2}$ per well of a 24 -well plate. After $24 \mathrm{~h}$, the cells were labeled with $2 \mu \mathrm{M}$ CellTracker Green CMFDA or CellTracker Red CMTPX according to the manufacturer's protocol. After washing the cells with PBS, we added $300 \mu \mathrm{l}$ of $0.125 \%$ trypsin-EDTA to detach the cells followed by $900 \mu \mathrm{l}$ of culture medium in each well. We collected $1.2 \mathrm{ml}$ of red-labeled cell suspension and $40 \mu \mathrm{l}$ of green-labeled cell suspension, which were mixed together (cell number ratio; red:green $=30: 1$ ). We added $5 \mathrm{ml}$ of culture medium to the mixture and centrifuged the cells. The cell pellet was resuspended in $150 \mu \mathrm{l}$ of culture medium and was seeded onto a collagen-coated glass-based dish $\left(0.5 \mathrm{~cm}^{2}\right)$. After $24 \mathrm{~h}$, images of the cells were captured every 6 min for $13 \mathrm{~h}$ or longer using a Nikon A1Rsi confocal microscope (Nikon, Tokyo, Japan), To confirm the lack of interference from each cell-labeling solution (CellTracker CMFDA and CMTPX), we stained the cells with Hoechst 33342 and took high-resolution images after time-lapse imaging.

Manual tracking of cells. We analyzed the images using ImageJ software (http://rsb.info.nih.gov/ij/). We used the LOCI Bio-Format plug-in (http://www.loci.wisc.edu/software/bio-formats) to process the acquired images and the Manual Tracking plug-in (http://rsbweb.nih.gov/ij/plugins/track/track.html) to track cell migration.

Tissue culture of the murine abdominal wall. Four- to ten-week-old C57BL/6 mice (Charles River Japan) were used. Their anterior abdominal walls were excised, washed with warm HBSS at least three times and incubated in culture medium $50 \%$ Opti-MEM, 25\% FBS-Gold, 24\% HBSS and 1\% antibiotic-antimycotic solution). The tissue was labeled using 5 or $10 \mu \mathrm{M}$ CellTracker Green CMFDA according to the manufacturer's protocol. After labeling, the tissue was subjected to time-lapse imaging using a Nikon A1Rsi confocal microscope. We took images of parietal mesothelial cells every $20 \mathrm{~min}$ for $10 \mathrm{~h}$ or longer. The motility of thirty or more cells was tracked from each sample by ImageJ.

Scanning electron microscopy. Immediately following dissection or after 1 day of incubation, the murine abdominal walls were fixed in phosphate buffer containing $2 \%$ glutaraldehyde for $30 \mathrm{~min}$. The samples were washed and fixed in $2 \%$ osmium tetroxide for $30 \mathrm{~min}$. After repeating the washing step, the samples were treated with $1 \%$ tannic acid for $30 \mathrm{~min}$ and fixed again using $2 \%$ osmium tetroxide for $30 \mathrm{~min}$. The tissues were dehydrated and coated with osmium tetroxide at a thickness of $10 \mathrm{~nm}$. We observed the samples using S-800S (Hitachi, Tokyo, Japan) scanning electron microscope.

TUNEL assay. Immediately following dissection or after $16 \mathrm{~h}$ of incubation, the murine abdominal walls were fixed in $10 \%$ neutral phosphate-buffered formalin. The tissues were subsequently sliced into sections, attached to slide glasses and deparaffinized. The specimens were washed with distilled water and subjected to antigen retrieval by protein digestion for $10 \mathrm{~min}$ at $37^{\circ} \mathrm{C}$. The specimens were washed with distilled water again and were incubated with $\mathrm{TdT}$ (Terminal deoxynucleotidyl transferase) for $50 \mathrm{~min}$ at $37^{\circ} \mathrm{C}$. $\mathrm{TdT}$ reaction solution was prepared by the addition of TdT solution (Wako Pure Chemical Industries, Osaka, Japan) and fluorescein12-dUTP (Roche Applied Science, Indianapolis, IN) to TdT reaction buffer (pH7.2, $30 \mathrm{mM}$ Tris- $\mathrm{HCl}, 140 \mathrm{mM}$ cacodylic acid, $1 \mathrm{mM} \mathrm{CoCl}_{2}, 140 \mathrm{mM} \mathrm{NaOH}$ ). The specimens were later washed with PBS and incubated with blocking solution (N101, NOF Corporation, Tokyo, Japan) for $30 \mathrm{~min}$ at room temperature. Followed by PBS washing, anti-fluorescein antibody conjugated with Alexa 488 and Hoechst 33342 were added to each slide. After $2 \mathrm{~h}$ incubation at $37^{\circ} \mathrm{C}$, the slides were washed again with PBS and subjected to inverted confocal microscopy. To make positive and negative controls, we incubated a slide for $30 \mathrm{~min}$ at $37^{\circ} \mathrm{C}$ with DNAse I solution (Invitrogen) before $\mathrm{TdT}$ reaction and incubated a slide without $\mathrm{TdT}$ enzyme during $\mathrm{TdT}$ reaction solution, respectively.

Pimonidazole-based evaluation of hypoxia. Murine anterior abdominal walls were prepared as described above. Hypoxyprobe $(200 \mu \mathrm{M})$ was added to each tissue immediately after tissue culture started or after $14 \mathrm{~h}$ incubation. After $2 \mathrm{~h}$ of incubation with hypoxyprobe, tissues were fixed and subjected to immunohistochemistry using anti-pimonidazole monoclonal antibody at a concentration of $1.4 \mu \mathrm{g} / \mathrm{mL}$. The tissues were observed using BZ8000 (Keyence, Japan)

Statistics. All experiments were performed three or more times. The unpaired Student's $t$-test was used to calculate each statistics. The statistics was calculated using Prism 5 (GraphPad Software Inc., San Diego, CA, USA).

1. Mutsaers, S. E. Mesothelial cells: their structure, function and role in serosal repair. Respirology 7, 171-191 (2002)

2. Herrick, S. E. \& Mutsaers, S. E. Mesothelial progenitor cells and their potential in tissue engineering. Int. J. Biochem. Cell Biol. 36, 621-642 (2004).

3. Andrews, P. M. \& Porter, K. R. The ultrastructural morphology and possible functional significance of mesothelial microvilli. Anat. Rec. 177, 409-426 (1973).

4. Fedorko, M. E. \& Hirsch, J. G. Studies on transport of macromolecules and small particles across mesothelial cells of the mouse omentum. I. Morphologic aspects. Exp. Cell Res. 69, 113-127 (1971).
5. Yung, S. \& Chan, T. M. Intrinsic cells: mesothelial cells - central players in regulating inflammation and resolution. Perit. Dial. Int. 29 Suppl 2, S21-27 (2009)

6. Van Der Wal, B. C., Hofland, L. J. Marquet, R. L., Van Koetsveld, P. M., Van Rossen, M. E. \& Van Eijck, C.H. Paracrine interactions between mesothelial and colon-carcinoma cells in a rat model. Int. J. Cancer 73, 885-890 (1997).

7. Hu, Q., Akatsuka, S., Yamashita, Y., Ohara, H., Nagai, H., Okazaki, Y., Takahashi, T. \& Toyokuni, S. Homozygous deletion of CDKN2A/2B is a hallmark of iron-induced high-grade rat mesothelioma. Lab. Invest. 90, 360-373 (2010).

8. Nagai, H., Okazaki, Y., Chew, S. H., Misawa, N., Yamashita, Y., Akatsuka, S., Ishihara, T., Yamashita, K., Yoshikawa, Y., Yasui, H., Jiang, L., Ohara, H. Takahashi, T., Ichihara, G., Kostarelos, K., Miyata, Y., Shinohara, H. \& Toyokuni, S. Diameter and rigidity of multiwalled carbon nanotubes are critical factors in mesothelial injury and carcinogenesis. Proc. Natl. Acad. Sci. USA 108, E1330-1338 (2011)

9. Akatsuka, S., Yamashita, Y., Ohara, H., Liu, Y. T., Izumiya, M., Abe, K., Ochiai, M., Jiang, L., Nagai, H., Okazaki, Y., Murakami, H., Sekido, Y., Arai, E., Kanai, Y., Hino, O., Takahashi, T., Nakagama, H. \& Toyokuni, S. Fenton reaction induced cancer in wild type rats recapitulates genomic alterations observed in human cancer. PLoS One 7, e43403 (2012).

10. Jiang, L., Akatsuka, S., Nagai, H., Chew, S. H., Ohara, H., Okazaki, Y., Yamashita, Y., Yoshikawa, Y., Yasui, H., Ikuta, K., Sasaki, K., Kohgo, Y., Hirano, S., Shinohara, Y., Kohyama, N., Takahashi, T. \& Toyokuni, S. Iron overload signature in chrysotile-induced malignant mesothelioma. J. Pathol. 228, 366-377 (2012).

11. Nagai, H. \& Toyokuni, S. Differences and similarities between carbon nanotubes and asbestos fibers during mesothelial carcinogenesis: shedding light on fiber entry mechanism. Cancer Sci. 103, 1378-1390 (2012).

12. Dobbie, J. W., Zaki, M. \& Wilson, L. Ultrastructural studies on the peritoneum with special reference to chronic ambulatory peritoneal dialysis. Scott. Med. J. 26, 213-223 (1981)

13. Di Paolo, S., Gesualdo, L., Ranieri, E., Grandaliano, G. \& Schena, F. P. High glucose concentration induces the overexpression of transforming growth factor-beta through the activation of a platelet-derived growth factor loop in human mesangial cells. Am. J. Pathol. 149, 2095-2106 (1996).

14. Ellis, $\mathrm{H}$. The cause and prevention of postoperative intraperitoneal adhesions. Surg. Gynecol. Obstet. 133, 497-511 (1971).

15. Nagai, H. \& Toyokuni, S. Biopersistent fiber-induced inflammation and carcinogenesis: lessons learned from asbestos toward safety of fibrous nanomaterials. Arch. Biochem. Biophys. 502, 1-7 (2010).

16. Mutsaers, S. E., Whitaker, D. \& Papadimitriou, J. M. Mesothelial regeneration is not dependent on subserosal cells. J. Pathol. 190, 86-92 (2000).

17. Whitaker, D. \& Papadimitriou, J. Mesothelial healing: morphological and kinetic investigations. J. Pathol. 145, 159-175 (1985).

18. Ryan, G. B., Grobety, J. \& Majno, G. Mesothelial injury and recovery. Am. J. Pathol. 71, 93-112 (1973).

19. Foley-Comer, A. J., Herrick, S. E., Al-Mishlab, T., Prele, C. M., Laurent, G. J. \& Mutsaers, S. E. Evidence for incorporation of free-floating mesothelial cells as a mechanism of serosal healing. J. Cell Sci. 115, 1383-1389 (2002).

20. Bolen, J. W., Hammar, S. P. \& Mcnutt, M. A. Reactive and neoplastic serosal tissue A light-microscopic, ultrastructural, and immunocytochemical study. Am. J. Surg. Pathol. 10, 34-47 (1986).

21. Pampinella, F., Roelofs, M., Castellucci, E., Chiavegato, A., Guidolin, D., Passerini-Glazel, G., Pagano, F. \& Sartore, S. Proliferation of submesothelial mesenchymal cells during early phase of serosal thickening in the rabbit bladder is accompanied by transient keratin 18 expression. Exp. Cell Res. 223, 327-339 (1996).

22. Mutsaers, S. E., Whitaker, D. \& Papadimitriou, J. M. Stimulation of mesothelial cell proliferation by exudate macrophages enhances serosal wound healing in a murine model. Am. J. Pathol. 160, 681-692 (2002).

23. Fotev, Z., Whitaker, D. \& Papadimitriou, J. M. Role of macrophages in mesothelial healing. J. Pathol. 151, 209-219 (1987).

24. Mutsaers, S. E. The mesothelial cell. Int. J. Biochem. Cell Biol. 36, 9-16 (2004).

25. Yap, A. S. \& Kovacs, E. M. Direct cadherin-activated cell signaling: a view from the plasma membrane. J. Cell Biol. 160, 11-16 (2003).

26. Hazan, R. B., Qiao, R., Keren, R., Badano, I. \& Suyama, K. Cadherin switch in tumor progression. Ann. N. Y. Acad. Sci. 1014, 155-163 (2004).

27. Hott, J. W., Sparks, J. A., Godbey, S. W. \& Antony, V. B. Mesothelial cell response to pleural injury: thrombin-induced proliferation and chemotaxis of rat pleural mesothelial cells. Am. J. Respir. Cell Mol. Biol. 6, 421-425 (1992).

28. Nasreen, N., Mohammed, K. A., Galffy, G., Ward, M. J. \& Antony, V. B. MCP-1 in pleural injury: CCR2 mediates haptotaxis of pleural mesothelial cells. Am. J. Physiol. -Lung Cell. Mol. Physiol. 278, L591-598 (2000).

29. Strukova, S. M. Thrombin as a regulator of inflammation and reparative processes in tissues. Biochemistry (Mosc). 66, 8-18 (2001).

30. Shireman, P. K., Contreras-Shannon, V., Ochoa, O., Karia, B. P., Michalek, J. E. \& Mcmanus, L. M. MCP-1 deficiency causes altered inflammation with impaired skeletal muscle regeneration. J. Leukoc. Biol. 81, 775-785 (2007).

31. Topley, N., Brown, Z., Jorres, A., Westwick, J., Davies, M., Coles G.A. \& Williams J.D. Human peritoneal mesothelial cells synthesize interleukin-8. Synergistic induction by interleukin-1 beta and tumor necrosis factor-alpha. Am. J. Pathol. 142, 1876-1886 (1993). 
32. Topley, N., Jorres, A., Luttmann, W., Petersen, M. M., Lang, M. J. Thierauch, K.H., Muller, C., Coles, G. A., Davies, M. \& Williams, J. D. Human peritoneal mesothelial cells synthesize interleukin-6: induction by IL-1 beta and TNF alpha. Kidney Int. 43, 226-233 (1993).

33. Eigenbrod, T., Park, J. H., Harder, J., Iwakura, Y. \& Nunez, G. Cutting edge: critical role for mesothelial cells in necrosis-induced inflammation through the recognition of IL-1 alpha released from dying cells. J. Immunol. 181, 8194-8198 (2008).

\section{Acknowledgements}

We thank Ikuyo Mizuguchi and Yoshikazu Fujita (Division for Medical Research Engineering, Nagoya University Graduate School of Medicine) for technical support. This study was supported by a Grant-in Aid from the Ministry of Education, Culture, Sports, Science and Technology of Japan, a MEXT grant (Special Coordination Funds for Promoting Science and Technology), Princess Takamatsu Cancer Research Fund (10-24213) and a Grant-in-Aid from the Japan Society for the Promotion of Science Fellows to H.N.

\section{Author contributions}

H.N. conceived the project, performed the experiments and wrote the paper. C.S.H. edited the manuscript and performed animal experiments. A.E., T.K., T.N., J.L. and S.A. gave conceptual advice. Y.O. and S.F. helped animal experiments. S.T. supervised the project and edited the manuscript.

\section{Additional information}

Supplementary information accompanies this paper at http://www.nature.com/ scientificreports

Competing financial interests: The authors declare no competing financial interests.

License: This work is licensed under a Creative Commons

Attribution-NonCommercial-NoDerivs 3.0 Unported License. To view a copy of this license, visit http://creativecommons.org/licenses/by-nc-nd/3.0/

How to cite this article: Nagai, H. et al. Metamorphosis of mesothelial cells with active horizontal motility in tissue culture. Sci. Rep. 3, 1144; DOI:10.1038/srep01144 (2013). 